\section{Fair-use policies aim to balance access and cost of publishing}

SIR - Your News story 'Openaccess policy flourishes at $\mathrm{NIH}^{\prime}$ (Nature 458, 690-691; 2009) raises the related question for publishers about the challenges inherent in providing the widest and most costeffective access to quality scientific literature.

At the non-profit American Institute of Physics, we (like other publishers) are concerned about the inescapable realities of accomplishing this aim. The reality is that publishing a quality journal costs money. The tradeoff is that those costs add value to a researcher's manuscript as it evolves from a draft submission to a final publication.

In our case, this transformation process requires peer review by tens of thousands of experts annually, as well as editing by postdoctoral physicists, refining of text and graphics, and use of increasingly sophisticated indexing and archiving measures. Government-mandated open-access policies will impose unintended negative consequences if they threaten the very business models that pay for quality publications.

Such mandates may not be necessary. We and other publishers are voluntarily developing copyright-friendly, fair-use policies that obviate the access issue. Harvard and the American Physical Society, for example, recently agreed on ways to facilitate authors' compliance with Harvard's new open-access policies when publishing in distinguished journals such as Physical Review, Physical Review Letters and Reviews of Modern Physics.

Even in the information age, maintaining the quality of the scientific literature costs money. How we can continue to improve access to scientific journals without compromising their quality is a question about which we should all be concerned. H. Frederick Dylla American Institute of Physics, One Physics Ellipse, College Park, Maryland 20740-3843, USA e-mail: dylla@aip.org

Nature Publishing Group (NPG) provides a free manuscript deposition service at http://tinyurl. $\mathrm{com} / \mathrm{mq} 4 m \times 2$, to enable authors to comply with funders' mandates. NPG's licence-to-publish policy is at http://tinyurl.com/4etxvz

\section{Funding ban could break careers at the toss of a coin}

SIR - In your News story 'UK scientists get funding ban reprieve' (Nature 459, 20; 2009), you report the response of the UK Engineering and Physical Sciences Research Council (EPSRC) to criticism of proposed changes to grant-submission eligibility. In my view, the EPSRC response still fails to address the central issue.

Even with the softened proposal, blacklisting will severely damage researchers' careers; moreover, it will selectively damage the productivity of our most innovative and daring researchers. It is critical that any measure used to do this should be robust, objective, transparent and widely trusted. Peer review as a means of numerically ranking grant proposals satisfies none of these criteria. Studies on peer review of grants (for example, S. Cole et al. Science 214, 881-886; 1981) and of papers (for example, L. Bornmann and H.-D. Daniel Learn. Publ. 22, 117-125; 2009) have consistently shown that peer review performs poorly at numerical ranking, that it is subject to serious random effects, and that it is not good at distinguishing between the majority of proposals and papers that fall between the very top and the very bottom. Correlation between results when a peer-review process is repeated is only marginally better than would be expected by chance.

Therefore, whether a proposal falls within the top or bottom $50 \%$ of the ranking is largely determined by chance, and has little relation to any objective measure of quality. The chief executive of the EPSRC has been reported as accepting that peer review is "basically a lottery" - a widely held view among researchers. However, the $50 \%$ mark is the key measure that the EPSRC proposes to use. The revised proposals are therefore still capable of potentially destroying people's careers on the basis of a measure that is only slightly better than flipping a coin.

Cameron Neylon Science and Technology Facilities Council, Rutherford Appleton Laboratory, Didcot OX11 0QX, UK, and School of Chemistry, University of Southampton, Southampton SO17 1BJ, UK e-mail: cameron.neylon@stfc.ac.uk

\section{Cancer screening for women in developing countries}

SIR - Your Editorial 'Early warnings' (Nature 458, 679; 2009) points out some pitfalls in the effectiveness of cancer screening. I would like to draw attention to problems associated with gynaecological screening in resource-poor countries.

Disease prevalence can cause a large variation in the positive predictive value (PPV) of tests whose sensitivity and specificity are comparable. If $10 \%$ of the population is likely to have a disease and 1,000 people are screened for it, then by using a test with $100 \%$ sensitivity and 99\% specificity, we should pick up 100 true positives and 10 false positives; the PPV will be 100 true positives to 110 total positives, or $91 \%$. But for a disease with an incidence of $0.1 \%$, a test of the same sensitivity and specificity will generate one true and 10 false positives per 1,000 screened, with a resultant PPV of one true positive/11 total positives, or $9 \%$.

Take, for example, screening tests for ovarian and cervical cancers, to identify precancerous conditions and early-stage disease. Although these cancers are widespread killers, they have a low incidence compared with, say, tuberculosis. Ovarian cancer has a worldwide incidence of $0.01 \%$ (International Agency for Research on Cancer, www-dep. iarc.fr), meaning that one screen, even with $99.9 \%$ specificity, will give a PPV of $9 \%$. The resulting surgery will be unnecessary for 10 out of 11 women who undergo it.

Such high specificity is very difficult to achieve, but anything lower is useless. Even multivariate systems combining many markers for ovarian cancer have failed to increase specificity above $99.9 \%$. Despite this wastefulness, sponsors and governments are under pressure to organize large-scale trials, as your Editorial mentions.

The value of screening is also affected by the feasibility of further investigations and treatment. Take cervical cancer, which overall is more than twice as common as ovarian cancer (although its incidence varies widely around the world): the position of the cervix means that even a low PPV will not undermine well-established screening programmes, as results can easily be verified by biopsy rather than a major operation.

However, in many resource-poor countries, where only about $1 \%$ of women may be screened for these cancers, the confounding false negatives and negative predictive values caused by low sensitivity present a big problem. Many cases will not be discovered until the disease is too far advanced for successful treatment. In such countries, huge populations and restricted resources mean that screening efforts may need to be abandoned in favour of inexpensive universal treatment or prophylaxis. Newer, targeted approaches for ovarian cancer (T. A. Yap et al. Nature Rev. Cancer 9, 167-181; 2009) and a cheaper vaccine for cervical cancer may prove helpful. Chinmoy K. Bose Gynaecological Oncology Section, Netaji Subhash Chandra Bose Cancer Research Institute, Kolkata 700 006, India e-mail: ckbose@hotmail.com 